\title{
PHASE-SPACE DYNAMIC TRACKING BY A TWO PICKUPS DATA ACQUISITION SYSTEM
}

\author{
Alessandro Drago, Maria Enrica Biagini, Susanna Guiducci, Catia Milardi, Miro Preger, Cristina \\ Vaccarezza, Mikhail Zobov (INFN / LNF, Frascati, Italy)
}

\section{Abstract}

A two pickups dynamic tracking data acquisition system has been developed at LNF for the DAФNE Phifactory. Two oscilloscopes sample horizontal and vertical sum and difference signals from two pickups simultaneously; the sampling clock is locked to the DAФNE timing system. A horizontal kick excites the beam motion and initiates the acquisition. Turn-by-turn signals are converted to beam position and stored on a server in a database using timestamp labels. Oscillation amplitude versus time, phase space distribution and frequency domain analysis have been evaluated for several lattices and different settings of sextupoles and octupoles. Results are used to check the DAФNE nonlinear model.

\section{INTRODUCTION}

DAФNE, the Frascati $\Phi$-Factory [1], is in operation since 1999. It produces $\mathrm{e}^{+} / \mathrm{e}^{-}$collisions at $1.02 \mathrm{GeV}$ c.m. energy, for three experiments: KLOE (kaon physics), FINUDA (nuclear physics)and DEAR (atomic physics), running one at a time. Non-linearities in the magnetic structures have been identified in sextupoles and wigglers.
A non-linear model [2] has been developed and a single BPM dynamic tracking acquisition system has been developed [3] to check it by recording turn-to-turn beam position. Recently this system has been upgraded to collect simultaneous signals from two beam position monitors in order to deliver the phase-space shape of the beam centroid motion.

The hardware and software schemes are described in the following section and examples of collected measurements are presented.

\section{SYSTEM DESCRIPTION}

The hardware scheme is plotted in Fig. 1. To study the phase-space behavior of the beam, a low current single bunch is stored in the main ring.

An oscillation of the bunch is excited by pulsing the injection (horizontal) kicker, and, at the same time, data acquisition is initiated. The system has 8 input channels collecting transverse difference and sum signals from two beam position monitors. Two oscilloscopes acquire these signals by sampling clocks delivered by the DA $\Phi$ NE Timing system [4] and synchronized before the measurements by means of a phase shifter module with two independent manual knobs.

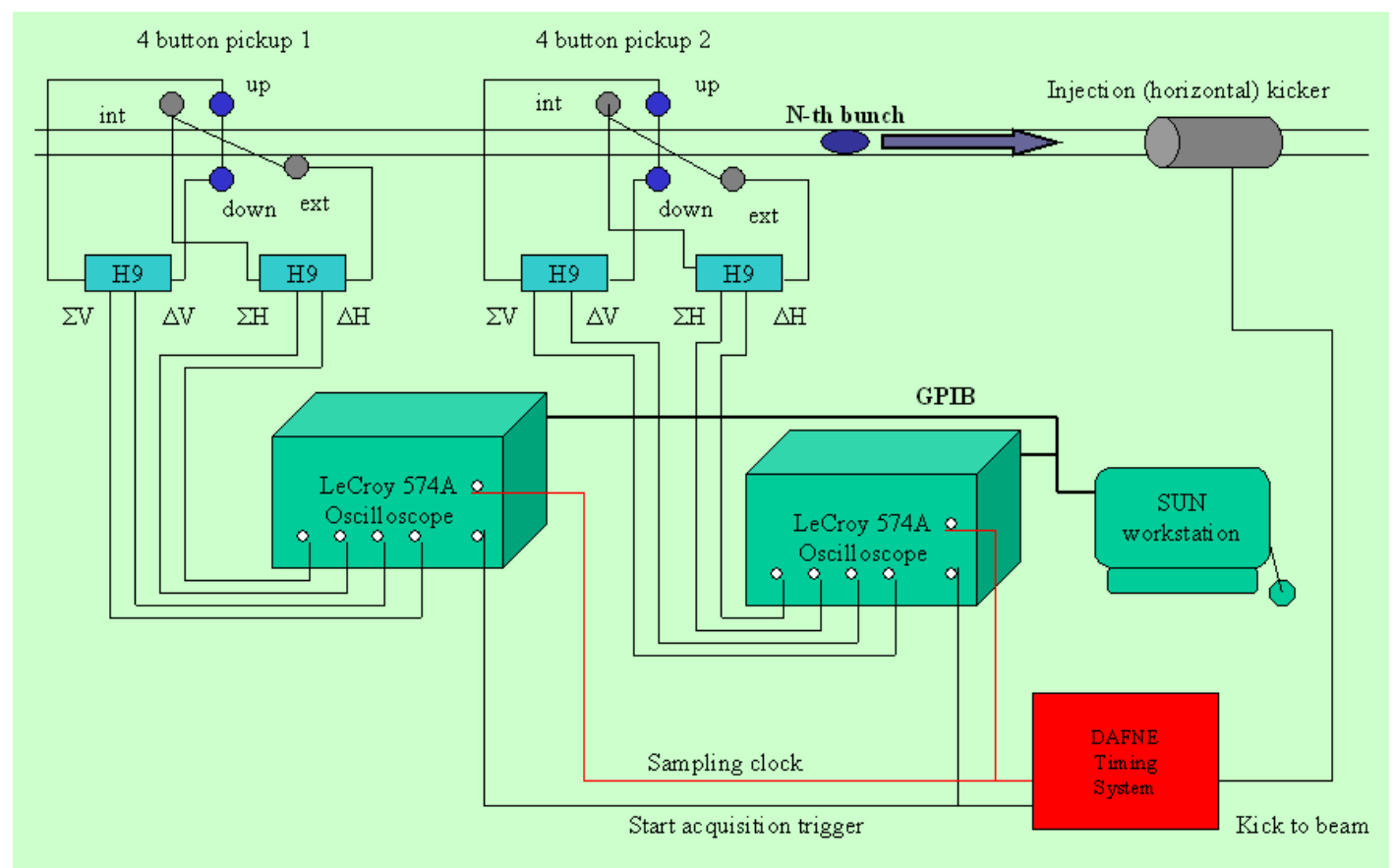

Fig. 1: Scheme of the setup used to record data from two beam position monitor: the beam is kicked by the injection kicker in the horizontal plane. 
The use of oscilloscopes instead of a multichannel sampler makes it easier to check if the timing is correct and if the transients are well acquired. However, there is an uncertainty of one turn on the alignment between data from the first oscilloscope and the second one, which have to be fixed offline by the user by the means of the software tools.

A SUN workstation downloads through an IEEE-488 bus the data from the oscilloscopes and several LABVIEW programs allow data storage and off-line analysis. Fig. 2 shows a directory tree ordering the files by particle type $\left(\mathrm{e}^{+}\right.$or $\left.\mathrm{e}^{-}\right)$date and time. Data in the files are in ASCII to be easily read by user programs or spreadsheets.

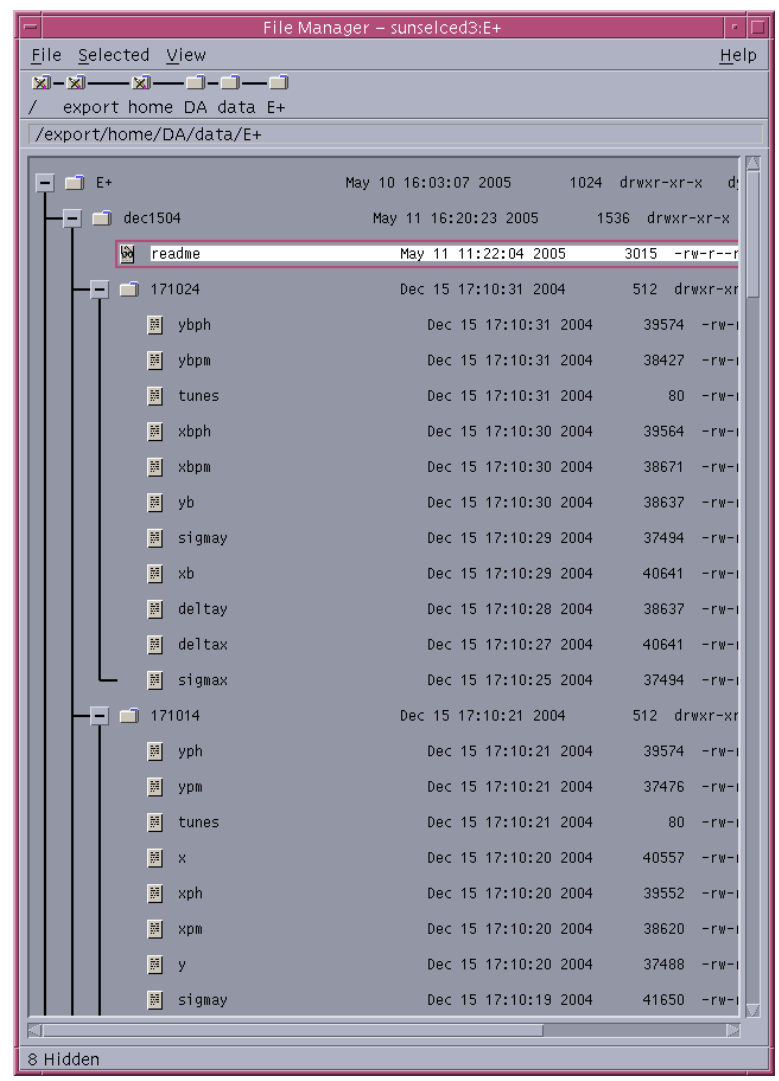

Figure 2: Directory tree and files stored by the data acquisition system.

The date folder contains a readme file, where the operator writes the relevant machine conditions, as well as beam current and kick amplitude. The date folder contains also the time folders with the measurement files describing the oscillation of the beam centroid. The files called sigmax, deltax, sigmay, deltay are rough data taken from the scopes in Volts. The files called $\mathrm{x}$ and $\mathrm{y}$ contain the calibrated horizontal and vertical displacements (in $\mathrm{mm}$ ) from the first BPM. Those called $\mathrm{xb}$ and $\mathrm{yb}$ are the same from the second one; $x p h, y p h, x b p h$ and $y b p h$ contain the corresponding Hilbert transforms of $x, y, x b$ and yb.
The two BPMs are rather close to each other in the ring. The betatron phase advance between them is $\approx 26$ degrees in both planes and the magnetic structure between the first BPM (BPBES108) and the second one (BPBES202) consists in two quadrupoles and three drift space without any non linear component. Therefore the betatron transfer matrix between the two BPMs is straightforward, and one can obtain the phase space trajectories at both monitors from the recorded beam positions at each turn.

Denoting by $M$ the transfer matrix between the two BPMs, $X_{1}$ the transverse position of the beam at the first monitor, $X^{\prime}{ }_{1}$ its angle with respect to the ideal trajectory (a straight line between the two monitors, $X_{2}$ and $X_{2}^{\prime}$ the corresponding quantities at the second monitor, we can write:

$$
\begin{aligned}
& X_{2}=M_{11} X_{1}+M_{12} X_{1}^{\prime} \\
& X_{2}^{\prime}=M_{21} X_{1}+M_{22} X_{1}^{\prime}
\end{aligned}
$$

from which we find the two angles corresponding to the two beam positions at each turn and therefore reconstruct the phase space trajectories at each monitor.

$$
\begin{gathered}
X_{1}^{\prime}=\frac{X_{2}-M_{11} X_{1}}{M_{12}} \\
X_{2}^{\prime}=\frac{\left(M_{12} M_{21}-M_{11} M_{22}\right) X_{1}+M_{22} X_{2}}{M_{12}}
\end{gathered}
$$

Fig. 3 and Fig. 4 show the phase trajectories of the beam at the two monitors. The dots are beam position and angle at each one of the first 50 revolutions after the kick, the line is the best ellipse fitting the measured data. Values of the optical functions at the monitor azimuth in the ring are derived from the fitting ellipse parameters.

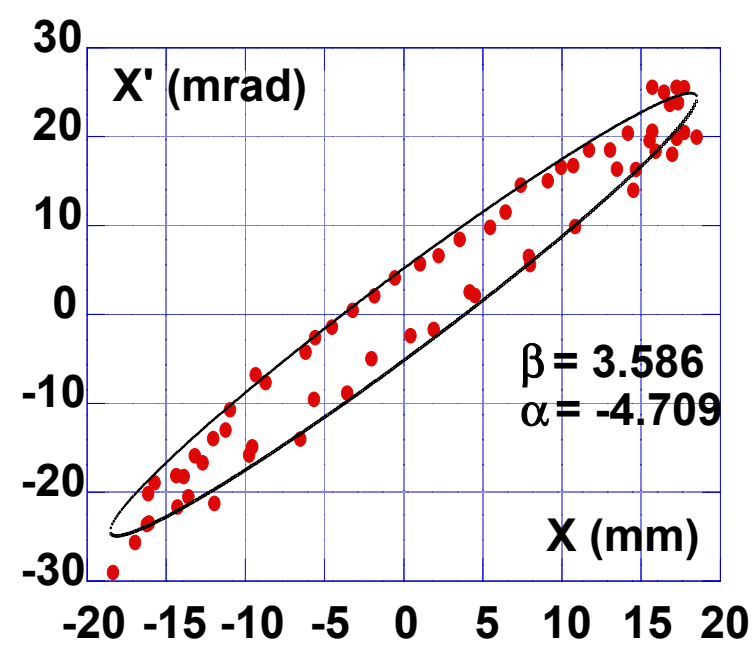

Figure 3: First 50 turns phase-space tracking after a kick at BPBES108. 


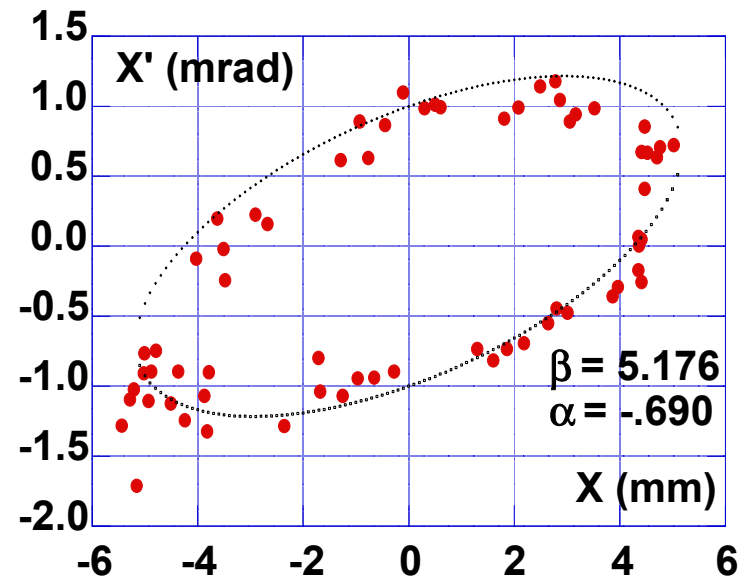

Figure 4: First 50 turns phase-space tracking after a kick at BPBES202

\section{MEASUREMENTS ON THE BEAM}

Measurements on the e+ ring have been performed at the end of 2004. In particular, on the last day of data acquisition, the positron ring has been characterized by a complete set of measurements for two different lattices: the regular "collision" lattice at and a second one with a slightly larger value of the momentum compaction.

For each lattice, the beam centroid position has been recorded for different kicker amplitudes, (from 10 to 20 $\mathrm{kV}$ ). with sextupoles on and octupoles on as in the normal operation lattice, with sextupoles on and octupoles off and with sextupole at low intensity and octupoles off. The case with sextupoles completely off was not recorded because even small amplitude kicks led to beam loss.

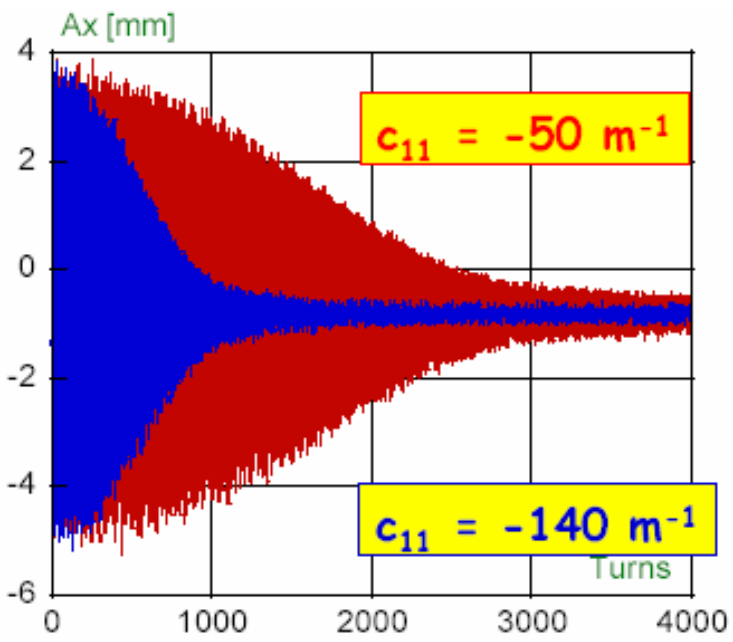

Figure 5: Beam decoherence: octupoles on (red) and off (blue).
An example of data analysis is plotted in Fig. 5, showing the horizontal displacements amplitude $\left(\mathrm{A}_{\mathrm{x}}\right)$ during successive revolutions. Beam decoherence is shown for a lattice with octupoles on (red) and off (blue). The $\mathrm{c}_{11}$ parameter correlating tune shift to oscillation amplitude can be estimated from the data.

By performing a Fourier transform of the recorded data it is possible to evaluate directly the tune shift versus oscillation amplitude for different lattices.

Fig.6 shows a comparison between the two cases of the previous figure. The oscillation amplitude is given in units of the beam transverse size. The vertical scale is the fractional part of the betatron tune.

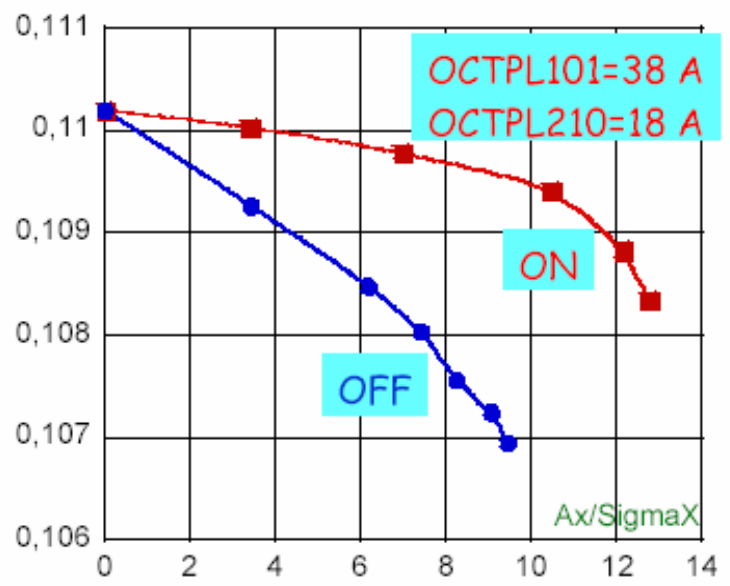

Figure 6: Tune shift versus amplitude: octupoles on (red) and off (blue).

\section{ACKNOWLEDGEMENTS}

We are grateful to to D.Pellegrini for the careful design of the two channels phase shifter module used to time the sampling clocks in input to the oscilloscopes.

\section{REFERENCES}

[1] M.Zobov et al., "DAФNE Operation and Plans for DAFNE2", ROAA001, this conference.

[2] C.Milardi et al., "Developments in Linear and NonLinear DAFNE Lattice", PAC2003, Portland, Oregon, May 12-16, 2003; LNF-03/012(P), 11/07/03.

[3] A.Drago, A.Stella, "The Dynamic Tracking Acquisition System for DAФNE e+/e- Collider", DIPAC2001, May 13-15, 2001, Grenoble, France.

[4] A.Drago et al., "Implementation and Performance of the DAФNE Timing System", 6th European Particle Accelerator Conference (EPAC 98), Stockholm, Sweden, 22-26 June 1998, p.1661, LNF-98/023 (P). 